\title{
Contribuição da análise de textura do núcleo celular para o diagnóstico diferencial de lesões foliculares da tireoide: comparação com marcadores imunoistoquímicos
}

\author{
Contribution of nuclear texture analysis for the \\ differential diagnosis of follicular lesions of the thyroid: \\ comparison to immunohistochemical markers
}

Rita C. Ferreira', Laura S. Ward ${ }^{2}$, Randall L. Adam ${ }^{3}$ Neucimar J. Leite ${ }^{3}$, Konradin Metze ${ }^{1}$, Patrícia S. de Matos ${ }^{1}$

${ }^{1}$ Departamento de Anatomia Patológica, Faculdade de Ciências Médicas (FCM), Universidade Estadual de Campinas (Unicamp), Campinas, SP, Brasil ${ }^{2}$ Departamento de Clínica Médica, FCM, Unicamp, Campinas, SP, Brasil ${ }^{3}$ Instituto de Computação, Unicamp, Campinas, SP, Brasil
Correspondência para: Patrícia S. de Matos Departamento de Anatomia Patológica, Unicamp Rua Tessália Viera de Camargo, 126 Caixa postal 6111, Barão Geraldo 13083-970 - Campinas, SP, Brasil pasmatos@fcm.unicamp.br

Recebido em 3/Fev/2009

Aceito em 12/Jul/2009

\section{RESUMO}

Objetivo e métodos: Com o propósito de investigar a contribuição do exame da cromatina nuclear no diagnóstico diferencial das lesões foliculares da glândula tireoide, foram estudadas 76 amostras previamente submetidas à análise de expressão proteica de HBME-1, CK-19 e galectina-3. Resultadlos: HBME-1 confirmou-se como o mais sensível marcador imunoistoquímico de malignidade. Uma série de variáveis morfométricas, densitométricas e de textura foram úteis na distinção entre os diferentes tipos de lesão folicular. Entre essas variáveis, o $r^{2}$, parâmetro relacionado à granularidade do núcleo, apresentou a melhor acurácia, sensibilidade, especificidade, valor preditivo positivo e negativo, diferenciando lesões benignas de malignas. Conclusão: A morfometria analítica de imagem da cromatina nuclear pode acrescentar acurácia ao diagnóstico diferencial das lesões de padrão folicular. Arq Bras Endocrinol Metab. 2009;53(7):804-10.

Descritores

Tireoide; diagnóstico diferencial; imunoistoquímica; cromatina

\begin{abstract}
Objective and methods: To investigate the utility of nuclear chromatin texture assessment in the differential diagnosis of follicular patterned lesions, by means of examining 76 samples previously submitted to the immunohistochemical protein analysis of HBME-1, CK-19 and galectina-3. Results: HBME-1 confirmed to be the most sensitive marker of malignancy. A series of morphometric, densitometric and texture variables were useful in the discrimination of the different types of follicular lesions. Among these variables, $r^{2}$, a parameter related to the granularity of the nucleus presented the best accuracy, sensitivity, specificity and positive and negative predictive values, distinguishing benign from malignant lesions. Conclusion: The morphometric analysis of nuclear chromatin images may add accuracy to the differential diagnosis of follicular patterned lesions. Arq Bras Endocrinol Metab. 2009;53(7):804-10.
\end{abstract}

Keywords

Thyroid; differential diagnosis; immunohistochemistry; chromatin

\section{INTRODUÇÃO}

$\mathrm{O}$ diagnóstico diferencial entre algumas lesões da tireoide é, muitas vezes, difícil, mesmo diante de amplas amostras do tecido. Em particular, o chamado "padrão folicular", um grupo que inclui nódulos ade- nomatosos ou hiperplásicos, adenomas foliculares, carcinomas foliculares e variantes foliculares dos carcinomas papilíferos, pode causar dificuldades diagnósticas mesmo aos patologistas mais experientes (1). Por outro lado, um diagnóstico histopatológico preciso é essencial 
para o planejamento clínico e cirúrgico e consequente prognóstico do paciente $(2,3)$. Métodos diagnósticos adicionais podem diminuir a ampla variação inter $e$ intraobservador do exame anatomopatológico, otimizando a decisão terapêutica (1).

Muitos marcadores de malignidade têm sido investigados, mas todos apresentam alguma desvantagem ou alguma limitação $(4,5)$. Galectina-3, HBME-1 ( $\mathrm{Hec}^{-}$ tor Battifora Mesothelial cell) e citoqueratina-19 (CK19) são os mais frequentemente utilizados na prática. Galectina-3 foi descrito como o mais sensível marcador diagnóstico para identificação pré-operatória de carcinomas da tireoide, com alta sensibilidade e especificidade em amostras histológicas e citológicas (6). Infelizmente, vários estudos imunoistoquímicos e moleculares não foram capazes de provar a utilidade da galectina-3 no padrão folicular, já que foi demonstrada sua expressão em tecidos tireoideanos normais e também nas lesões benignas (7-9). O HBME-1 foi descrito como útil no diagnóstico de tumores foliculares (10-12). Infelizmente, o HBME-1 parece se expressar com menor intensidade em lesões com células de Hürthle ou alterações apócrinas, frequentes nas lesões foliculares (12). Um terceiro marcador, CK-19, também pode ser útil no diagnóstico da variante folicular do carcinoma papilífero por sua forte e difusa positividade citoplasmática (13). No entanto, nosso grupo, e outros, demonstrou que esses três marcadores têm sensibilidade e especificidade limitadas, mesmo quando usados em conjunto $(14,15)$.

Estudos de morfometria nuclear vêm sendo empregados há mais de 20 anos no sentido de conferir maior precisão ao diagnóstico anatomopatológico quantificando objetivamente características morfológicas (16-18). Vários estudos morfonucleares por análise de imagem digital têm sido aplicados para análise do aspecto nuclear e do padrão de distribuição de cromatina em diversas neoplasias, incluindo tireoidianas (19-22). Como alterações do metabolismo celular influem diretamente na distribuição da cromatina, o estudo da textura nuclear permite detectar pequenas alterações que não são óbvias ao olho humano (23-26). Um estudo preliminar de nosso grupo sugeriu que alguns parâmetros morfométricos poderiam auxiliar no diagnóstico diferencial de lesões foliculares (16).

O presente estudo teve como objetivo ampliar o estudo anterior, verificando a utilidade da análise de imagem computadorizada na discriminação entre lesões foliculares benignas e malignas da tireoide e comparar os resultados àqueles obtidos por meio de estudo imunoistoquímico com os marcadores HBME-1, galectina-3 e CK-19.

\section{MÉTODOS}

Foram selecionados 76 blocos de lesões de padrão folicular da glândula tireoide incluindo 12 nódulos adenomatosos (NA), 18 adenomas foliculares (AF), 24 carcinomas foliculares minimamente invasores (CFMI) e 22 variantes foliculares do carcinoma papilífero (VFCP), todos previamente estudados por imunoistoquímica com galectina-3, HBME-1 e CK-19 (14).

A digitalização das imagens foi realizada com o auxílio do fotomicroscópio Axiophot para campo claro, contraste e epifluorescência (Carl Zeiss), com a objetiva de 100 aumentos Achroplan de imersão em óleo, acoplado ao sistema de câmara Axiophot e ao software para aquisição de imagens KS-300. A análise de imagem digitalizada foi realizada após a aquisição de 100 núcleos da periferia da lesão (somando um total de 7600 núcleos). Cada imagem nuclear foi submetida à segmentação semi-interativa. Foram adquiridos núcleos sem sobreposição de qualquer estrutura, por exemplo, de outros núcleos. As imagens foram capturadas no foco em que a maior parte dos contornos nucleares estava nítida e somente foram adquiridos núcleos de células identificadas como foliculares, desprezando-se quaisquer outras, como exemplificado nas figuras 1 e 2. Considerando-se que a imagem digital de um núcleo é composta por pixels com graus de cinza formando um padrão, a textura nuclear depende da relação espacial de cada pixel com os pixels vizinhos, podendo-se utilizar modelos matemáticos para a sua avaliação (21). Com auxílio de um software, os núcleos segmentados foram transformados em imagens com diferentes graus de cinza $(0$ a 255$)$.

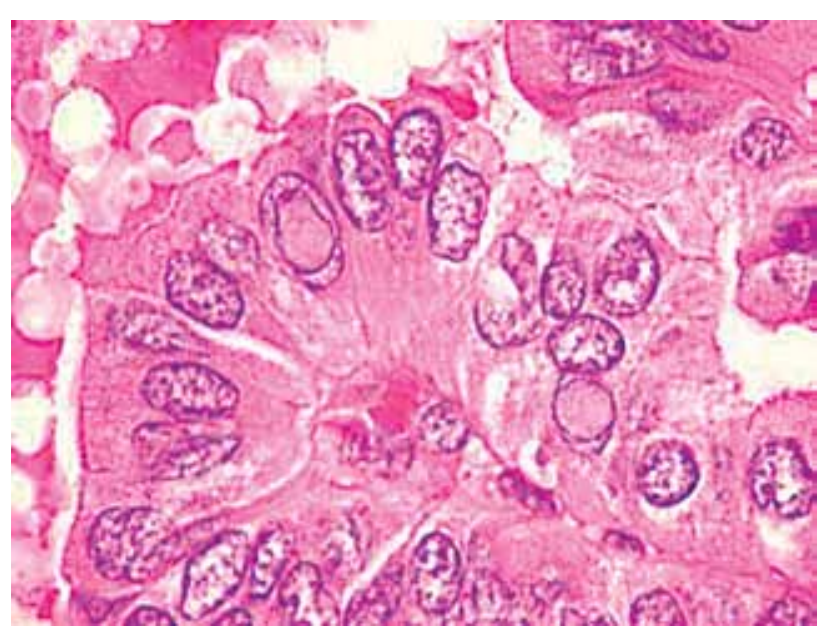

Figura 1. Exemplo de imagem digitalizada de variante folicular do carcinoma papilifero (VFCP) adquirida pelo sistema fotográfico empregado (HE 100x). 


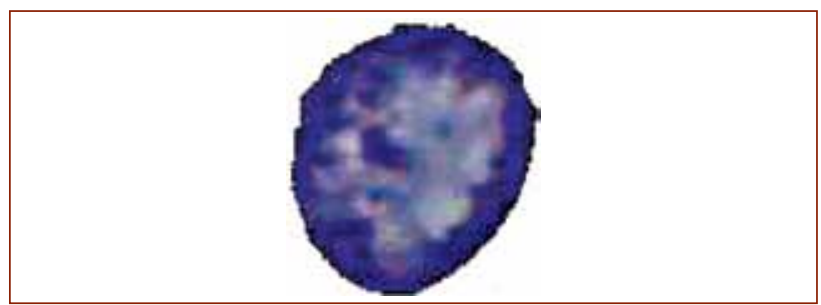

Figura 2. Exemplo de núcleo segmentado adquirido pelo sistema fotográfico empregado (HE 100x).

Avaliaram-se três grupos de variáveis na textura de cada núcleo: descritores morfométricos; densitométricos clássicos e descritores de matriz de coocorrência, incluindo a dimensão fractal e o seu "goodness of fit”. Esses parâmetros foram comparados às expressões quantificadas de acordo com o número de células e a intensidade da coloração imunoistoquímica das proteínas HBME-1, CK-19 e galectina-3, conforme anteriormente descrito (14).

O equipamento utilizado e o software correspondente pertencem ao Departamento de Anatomia Patológica da Faculdade de Ciências Médicas da Unicamp, local onde o trabalho foi realizado.

\section{Variáveis morfométricas}

Entre os descritores morfométricos, o fator de forma (FF) nos permite avaliar quão próxima a forma do contorno da imagem segmentada é da circunferência. Avalia a integridade do núcleo e, quanto mais próximo da forma arredondada, mais o FF se aproxima de 1 . Quanto mais distante da forma arredondada (por alongamento ou irregularidade no contorno), mais o fator de forma se torna próximo de zero.

Entre as variáveis morfométricas obtidas da análise de imagem nuclear, utilizou-se o percentil 5 do fator de forma.

\section{Variáveis densitométricas}

Estas variáveis analisam quantitativamente a densidade da cromatina nuclear. A relação entre os graus de cinza em uma imagem digital demonstram áreas de baixa, média ou alta condensação da cromatina, o que nos permite avaliar a densidade.

A densidade óptica (DO) é uma variável que se refere à intensidade de luz que é transmitida após sua passagem pelo tecido em relação à intensidade de luz que incide sobre a imagem nuclear. A quantidade de cromatina em qualquer ponto da imagem nuclear é proporcional à densidade óptica.
As variáveis matemáticas utilizadas na análise de variáveis densitométricas incluem o grau de cinza mínimo (GLevMin) e a média da densidade óptica (mDO).

\section{Variáveis de coocorrência}

As variáveis derivadas da matriz de coocorrência são calculadas a partir da relação entre os pixels em uma imagem convertida em graus de cinza e descrevem o quanto uma dada possibilidade de relação entre os pixels se repete na imagem (15).

A energia ("Energy") é uma variável que designa regiões de intensa uniformidade nuclear em certos pontos da imagem com relação aos graus de cinza. Assim, quanto menor a uniformidade entre os graus de cinza, menor é a energia, enquanto grande homogeneidade entre os graus de cinza descreve alta energia. Calculou-se o percentil 5 da energia.

Entre os modelos matemáticos que permitiram obter dados das variáveis de coocorrência, utilizou-se o percentil 95 para cálculo da tonalidade (cluster shade) e da proeminência (cluster proeminence) dos agrupamentos. Essas variáveis representam medidas de condensação da cromatina nuclear.

O parâmetro "goodness-of-fit" ou r $\mathrm{r}^{2}$, também denominado grau de aproximação da dimensão fractal de Minkowski-Bouligand, avalia o grau de granularidade da imagem nuclear.

Os dados foram comparados por análise de variância (nível de significância $\alpha<0,05$ ) com uso do software WinStat 3.1.

Este trabalho foi desenvolvido após aprovação pelo Comitê de Ética em Pesquisa da Faculdade de Ciências Médicas da Universidade Estadual de Campinas sob parecer $n^{\circ} 388 / 2005$.

\section{RESULTADOS}

A tabela 1 resume os dados de positividade para os marcadores imunoistoquímicos empregados: HBME-1, CK-19 e galectina-3.

Os resultados mais relevantes selecionados da análise da imagem nuclear são descritos a seguir.

\section{Variáveis morfométricas}

A variável P5FF mostrou diferença significativa entre adenoma folicular $(\mathrm{AF})$ e a variante folicular do carcinoma papilífero (VFCP), além de diferenciar o carcinoma folicular minimamente invasivo (CFMI) da VFCP (Gráfico l). 
Tabela 1. Positividade imunoistoquímica do HBME-1, CK-19 e galectina-3 em lesões foliculares da tireoide: nódulo adenomatoso (NA); adenoma folicular (AF); variante folicular do carcinoma papilífero (VFCP); carcinoma folicular minimamente invasor (CFMI). 0 padrão de positividade foi considerado: difuso (D) ou focal (F), sendo este último subdividido em focal segmentar (Fs) ou focal celular (Fc) de acordo com o método anteriormente descrito (11)

\begin{tabular}{ccccccc}
\hline \multirow{2}{*}{$\begin{array}{c}\text { Diagnóstico } \\
\text { (total casos) }\end{array}$} & \multicolumn{3}{c}{ Positividade (\%) } & \multicolumn{3}{c}{ Padrão de positividade predominante (\%) } \\
\cline { 2 - 7 } & HBME-1 & CK-19 & galectina-3 & HBME-1 & CK-19 & galectina-3 \\
\hline NA (12) & $4(33,3)$ & $2(16,7)$ & $1(8,4)$ & FC $(75)$ & FC $(100)$ & FC $(100)$ \\
AF (18) & $10(55,6)$ & $6(33)$ & $2(11)$ & Fs $(66,7)$ & Fs $(66,7)$ & FC $(100)$ \\
VFCP (22) & $18(72)$ & $11(37)$ & $11(37)$ & D $(77,5)$ & D $(65,2)$ & D $(65,2)$ \\
CFMI (24) & $15(39,4)$ & $5(13,1)$ & $5(13,1)$ & D $(31,3)$ & Fs $(31,3)$ & Fs $(31,3)$ \\
\hline
\end{tabular}

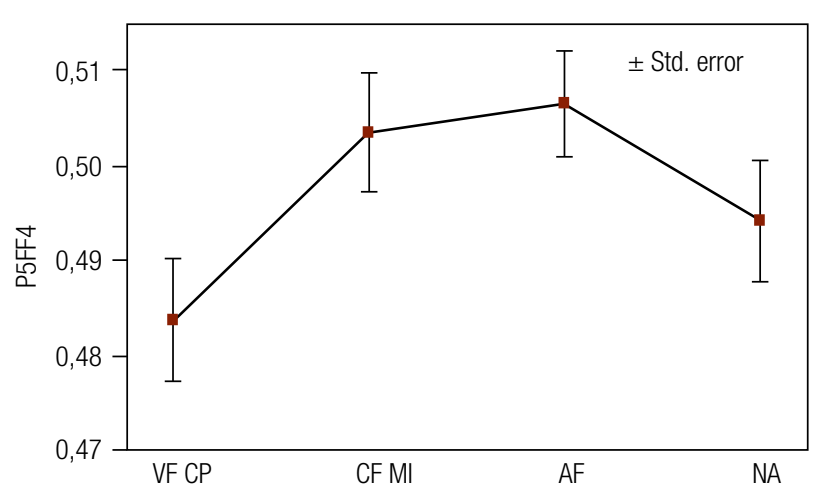

Gráfico 1. Comparação entre nódulo adenomatoso (NA), adenoma folicular (AF), carcinoma folicular minimamente invasor (CFMI) e a variante folicular do carcinoma papilifero (VFCP) utilizando o quinto percentil do fator de forma (P5FF).

\section{Variáveis densitométricas}

As variáveis densitométricas estudadas demonstraram diferenças significativas entre nódulo adenomatoso (NA) e AF; AF e CFMI, AF e VFCP, NA e CFMI e entre CFMI e VFCP. Entre todas as variáveis, a média do GLevMin (grau de cinza mínimo), representada no gráfico 2, distingue de forma clara os $\mathrm{NA}$ dos $\mathrm{AF}$ e os AF das VFCP.

A média da densidade óptica $(\mathrm{mDO})$ diferenciou NA de AF, e AF de VFCP (Gráfico 3).

\section{Variáveis de coocorrência}

São apresentados, a seguir, gráficos das variáveis derivadas da matriz de coocorrência que melhor discriminaram os grupos de lesões foliculares estudadas. $\mathrm{O}$ quinto percentil da "energia" (P5Energy), apresentado no Gráfico 4, aponta diferença significativa entre todos os subgrupos, exceto NA e VFCP, mas representa a variável que obteve o melhor desempenho em discriminar os quatro grupos estudados. O percentil 95 da tonalidade dos agrupamentos (P95CluShade) mostrou diferença significante entre todas as lesões foliculares (Gráfico 5). Observou-se que o percentil 95 da proeminência dos grupamentos (P95CluProem) diferenciou $\mathrm{NA}$ e AF, NA e CFMI, AF e VFCP, e CFMI e VFCP (Gráfico 6). $A$ variável $r^{2}$ mostrou diferença significativa entre NA e CFMI, NA e VFCP, AF e CFMI e entre AF e VFCP (Gráfico 7).

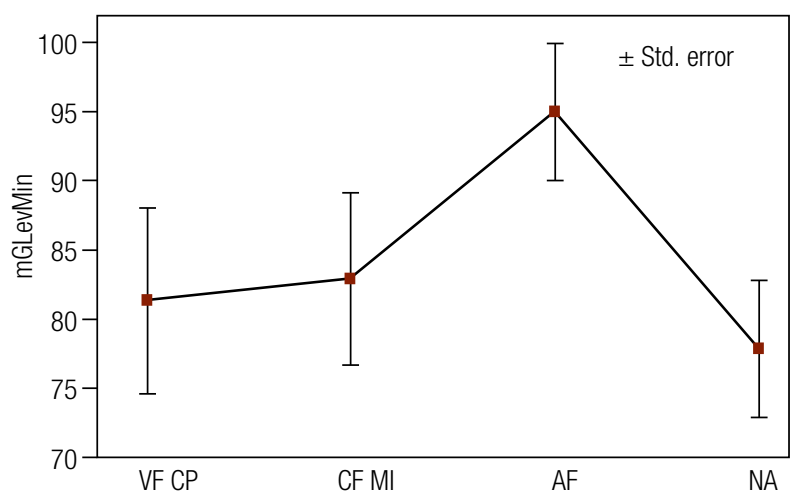

Gráfico 2. Comparação entre nódulo adenomatoso (NA), adenoma folicular (AF), carcinoma folicular minimamente invasor (CFMI) e a variante folicular do carcinoma papilifero (VFCP) utilizando a média do grau de cinza mínimo (GLevMin).

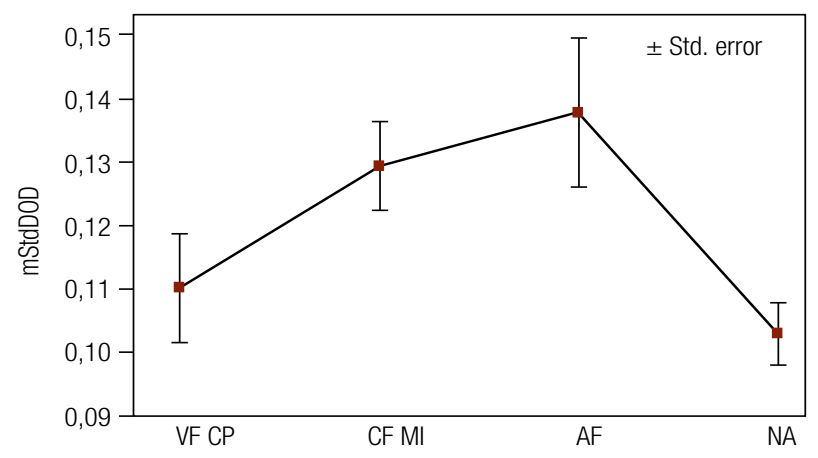

Gráfico 3. Comparação entre nódulo adenomatoso (NA), adenoma folicular (AF) carcinoma folicular minimamente invasor (CFMI) e a variante folicular do carcinoma papilífero (VFCP) utilizando a média da densidade óptica (mD0). 


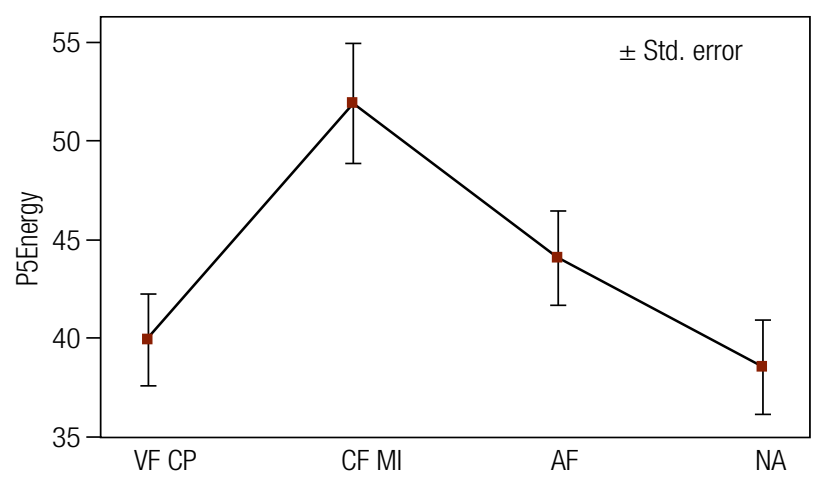

Gráfico 4. Comparação entre nódulo adenomatoso (NA), adenoma folicular (AF), carcinoma folicular minimamente invasor (CFMI) e a variante folicular do carcinoma papilifero (VFCP) utilizando o quinto percentil da "energia" (P5Energy).

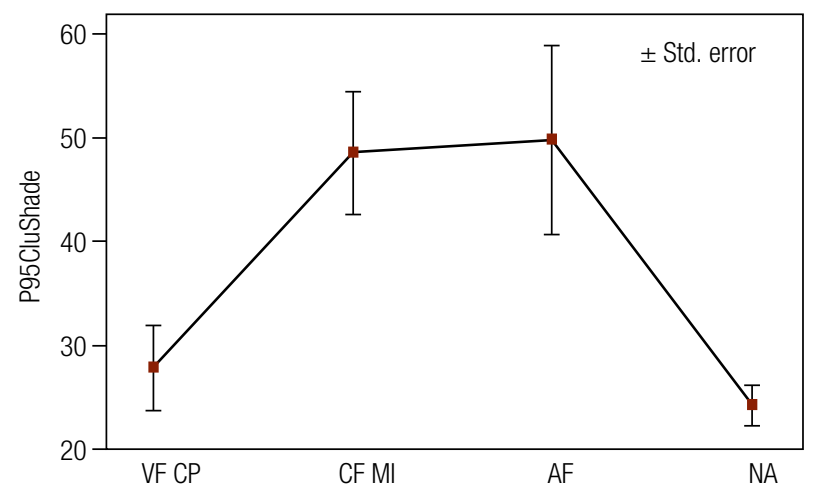

Gráfico 5. Comparação entre nódulo adenomatoso (NA), adenoma folicular (AF), carcinoma folicular minimamente invasor (CFMI) e a variante folicular do carcinoma papilifero (VFCP) utilizando o percentil 95 da tonalidade dos agrupamentos (P95CluShade).

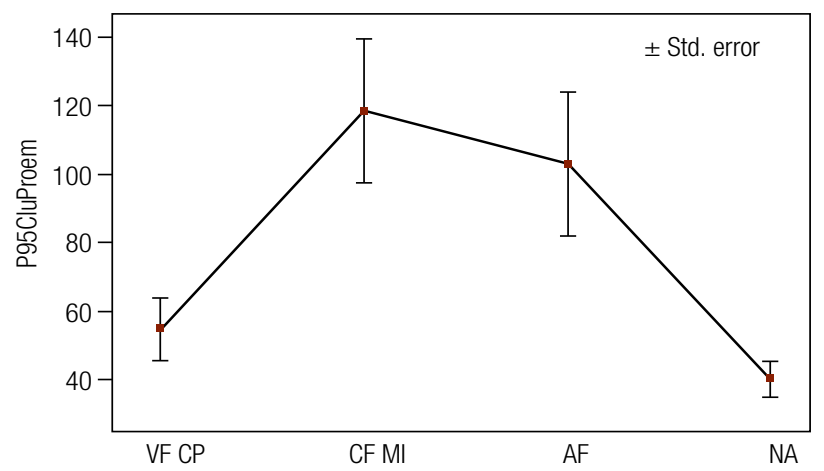

Gráfico 6. Comparação entre nódulo adenomatoso (NA), adenoma folicular (AF), carcinoma folicular minimamente invasor (CFMI) e a variante folicular do carcinoma papilifero (VFCP) utilizando o percentil 95 da proeminência dos agrupamentos (P95CluProem).

Não houve relação entre os valores quantificados da imunoistoquímica para qualquer um dos marcadores estudados com as variáveis de textura nuclear analisadas.

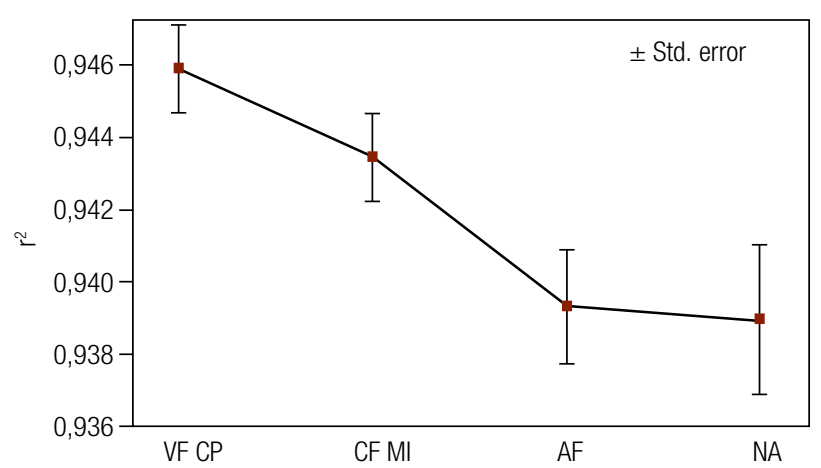

Gráfico 7. Comparação entre nódulo adenomatoso (NA), adenoma folicular (AF), carcinoma folicular minimamente invasor (CFMI) e a variante folicular do carcinoma papilifero (VFCP) utilizando a "Best $R$-square of regression fractal dimension of Minkowski-Bouligand ( $\left.\mathbf{r}^{2}\right)$ ".

A tabela 2 resume a utilidade clínica de todos os parâmetros estudados. Observou-se que os melhores resultados em termos de acurácia, sensibilidade, especificidade, valor preditivo positivo e negativo foram obtidos com a marcação com HBME-l e com a variável morfométrica $\mathrm{r}^{2}$.

Tabela 2. Acurácia, sensibilidade, especificidade, valor preditivo positivo e negativo para as variáveis (\%) na discriminação entre lesões benignas e malignas da tireoide

\begin{tabular}{|c|c|c|c|c|c|}
\hline Variável & Acurácia & Sensibilidade & Especificidade & $\begin{array}{c}\text { Valor } \\
\text { preditivo } \\
+\end{array}$ & $\begin{array}{c}\text { Valor } \\
\text { preditivo } \\
-\end{array}$ \\
\hline$r^{2}$ & 71,05 & 73,91 & 66,66 & 77,27 & 62,50 \\
\hline$\%$ HBME-1 & 68,42 & 56,52 & 86,66 & 86,67 & 56,52 \\
\hline mGLevMin & 59,21 & 56,52 & 63,33 & 70,27 & 48,71 \\
\hline P5Energy & 56,58 & 50,00 & 66,66 & 69,69 & 46,51 \\
\hline \% galectina-3 & 56,57 & 28,26 & 100,00 & 100,00 & 47,62 \\
\hline \% CK-19 & 55,26 & 26,09 & 100,00 & 100,00 & 46,88 \\
\hline P95CluProem & 51,32 & 36,96 & 53,33 & 62,16 & 41,03 \\
\hline P5FF4 & 50,00 & 52,17 & 46,66 & 60,00 & 38,89 \\
\hline P95CluShade & 47,37 & 60,87 & 26,66 & 56,00 & 30,77 \\
\hline mStdDOD & 53,95 & 65,22 & 36,66 & 61,22 & 29,73 \\
\hline
\end{tabular}

\section{DISCUSSÃO}

A popularização da ultrassonografia e da punção aspirativa por agulha fina, métodos simples, de custo relativamente baixo, de ampla disponibilidade e grande sensibilidade, aumentou muito o número de casos suspeitos de câncer de tiroide encaminhados ao patologista $(4,5,28)$. $\mathrm{O}$ câncer diferenciado da tiroide é o tumor cuja incidência mais cresceu entre os anos de 1992 e 2002 nos Estados Unidos, particularmente à custa de tumores pe- 
quenos, atingindo índice de 6,3\% ao ano entre 1997 e 2003 [Surveillance, Epidemiology, and End Results Program (SEER); dados disponíveis no site da American Cancer Society]. Registros nacionais de câncer [Instituto Nacional de Câncer (Inca); dados dos registros de base populacional] e publicações brasileiras mostram que um fenômeno semelhante vem ocorrendo no Brasil.

O diagnóstico anatomopatológico preciso é fundamental para descartar malignidade e também para o planejamento terapêutico e consequente prognóstico do paciente com câncer da tiroide $(3,4)$. Infelizmente, mesmo entre especialistas em patologia tireoidiana, o grau de concordância intra e interobservador beira os $20 \%$ nas lesões de padrão folicular $(1,29)$. Assim, ferramentas auxiliares ao diagnóstico, como a imunoistoquímica e a morfometria (16), têm sido buscadas.

Usando lesões previamente estudadas com auxílio da galectina-3, HBME e CK-19, demonstrou-se, no presente estudo, que alguns parâmetros morfométricos que incluem variáveis morfométricas, densitométricas e de coocorrência podem acrescentar precisão ao diagnóstico histopatológico.

Entre as variáveis morfométricas, o FF conseguiu distinguir lesões benignas de malignas (AF e VFCP) e mostrou diferença entre lesões malignas de padrão folicular, como o CFMI e a VFCP. Realmente, o FF se correlaciona com o tamanho e a variação de forma dos núcleos, características essenciais que também podem auxiliar no diagnóstico citológico obtido por punção aspirativa realizada com agulha fina (30).

As variáveis densitométricas foram úteis na distinção entre lesões benignas e malignas, como AF de VFCP. A variável densitométrica mGLevMin (média do grau de cinza mínimo) apresentou diferenças significativas entre lesões benignas e malignas, o que também era esperado, já que essa variável ressalta os aspectos nucleares de distribuição periférica e irregular da cromatina nuclear observados em casos de VFCP, quando se comparam aos núcleos hipercromáticos com cromatina mais densa e uniforme dos $\operatorname{AF}$ (30). Outra variável que se destacou nessa análise foi o P5Energy, que se mostrou menor na VFCP, demonstrando que nesta lesão o núcleo tem uma menor organização da cromatina. Pode-se fazer um paralelo com os aspectos identificados à microscopia óptica, na qual a cromatina é "salpicada" e periférica, colaborando para o aspecto nuclear claro da VFCP (14).

Entre as viáveis de coocorrência, observamos que o $r^{2}$ diferencia lesões benignas de malignas, confirmando nossos dados preliminares anteriormente publicados $\mathrm{e}$ sugerindo que quanto maior a granularidade da cromatina, maiores as alterações observadas em células neoplásicas malignas (16).

A comparação de acurácia dos parâmetros utilizados na morfometria com os imunoistoquímicos já rotineiramente empregados na caracterização das lesões de padrão folicular e no diagnóstico de malignidade em nosso serviço indica que a técnica de análise de imagem pode ser muito útil. Na verdade, essa técnica poderá ser ainda mais útil em células obtidas por punção aspirativa com agulha fina, cujo núcleo é mais facilmente isolado do que em tecido, pois as células estão mais esparsas no esfregaço.

Conclui-se que a análise morfométrica, que depende de simples incorporação de um software à análise digital de imagem já realizada em laboratórios de patologia, pode acrescentar acurácia à análise morfológica clássica e à imunoistoquímica na avaliação de lesões de padrão folicular.

Agradecimentos: os autores L.S.W e K.M são pesquisadores do Conselho Nacional de Desenvolvimento Científico e Tecnológico $(\mathrm{CNPq})$. Este trabalho recebeu apoio financeiro da Coordenação de Aperfeiçoamento de Pessoal de Nível Superior (Capes), do Fundo de Apoio ao Ensino, à da Pesquisa e à Extensão (Faepex), da Fundação de Amparo à Pesquisa do Estado de São Paulo (Fapesp) e do CNPq.

Declaração: os autores declaram não haver conflitos de interesse científico neste estudo.

\section{REFERÊNCIAS}

1. Franc $B$, De La Salmonière $P$, Lange $F$, Hoang $C$, Louvel $A$, de Roquancourt $A$, et al. Interobserver and intraobserver reproducibility in the histopathology of follicular thyroid carcinoma. Hum Pathol. 2003;34(11):1092-100.

2. Ward LS, Valente HOF, de Araujo PPC, Tincani AJ, Assumpcão LVM. Commonly used prognostic scoring systems are not adequate to predict the outcome of papillary microcarcinomas of the thyroid. Pediatric Blood \& Cancer. 2008;50(6):1288-9.

3. Fagin JA, Ward LS, Kimura ET. Câncer de tireóide: passado, presente e futuro. Arq Bras Endocrinol Metab. 2007;51(5):641-2.

4. Cerutti JM. Nodule diagnosed as follicular patterned lesion: Are biomarkers the promise? Arq Bras Endocrinol Metabol. 2007;51(5):832-42.

5. Meyer ELS, Wagner MS, Maia AL. lodothyronine deiodinases expression in thyroid neoplasias. Arq Bras Endocrinol Metabol. 2007;51(5):690-700.

6. Bartolazzi A, Gasbarri A, Papotti M, Bussolat G, Lucante T, Khan A, et al. Thyroid Cancer Study Group. Application of an immunodiagnostic method for improving preoperative diagnosis of nodular thyroid lesions. Lancet. 2001;357(9269):1644-50.

7. Martins L, Matsuo SE, Ebina KN, Kulcsar MA, Friguglietti CV, Kimura ET. Galectin-3 messenger ribonucleic acid and protein are expressed in benign thyroid tumors. J Clin Endocrinol Metab. 2002;87(8):4806-10. 
8. Feilchenfeldt J, Totsch M, Sheu SY, Robert J, Spiliopoulos A, Frilling $A$, et al. Expression of galectin-3 in normal and malignant thyroid tissue by quantitative PCR and immunohistochemistry. Mod Pathol. 2003;16(11):1117-23.

9. Jakubiak-Wielganowicz M, Kubiak R, Sygut J, Pomorski L, Kordek R. Usefulness of galectin-3 immunohistochemistry in differential diagnosis between thyroid follicular carcinoma and follicular adenoma. Pol J Pathol. 2003;54(2):111-5.

10. Miettinen $M$, Karkkainen P. Differential reactivity of HBME-1 and CD15 antibodies in benign and malignant thyroid tumours. Preferential reactivity with malignant tumours. Virchows Arch. 1996;429(4-5):213-9.

11. Sack MJ, Astengo-Osuna $C$, Lin BT, Battifora H, Livolsi VA. HBME-1 immunostaining in thyroid fine-needle aspirations: a useful marker in the diagnosis of carcinoma. Mod Pathol. 1997;10(7):668-74.

12. Mai KT, Bokhary R, Yazdi HM, Thomas J, Commons AS. Reduced HBME-1 immunoreactivity of papillary thyroid carcinoma and papillary thyroid carcinoma-related neoplastic lesions with Hurthle cell and/or apocrine-like changes. Histopathology. 2002;40(2):133-42.

13. Beesley MF, McLaren KM. Cytokeratin 19 and galectin-3 immunohistochemistry in the differential diagnosis of solitary thyroid nodules. Histopathology. 2002;41(3):236-43.

14. Matos PS, Ferreira AP, de Oliveira Facuri F, Assumpção LV, Metze K, Ward LS. Usefulness of HBME-1, cytokeratin 19 and galectin-3 immunostaining in the diagnosis of thyroid malignancy. Histopathology. 2005;47(4):391-401.

15. Asa SL. The role of immunohistochemical markers in the diagnosis of follicular-patterned lesions of the thyroid. Endocr Pathol. 2005;16(4):295-309.

16. Ferreira RC, Matos PS, Adam RL, Leite NL, Metze K. Application of the Minkowski-Bouligand fractal dimension for the differential diagnosis of thyroid follicular neoplasias. Cell Oncol. 2006;28(56):331-3.

17. Metze K, Adam RL, Silva PV, Carvalho RB, Leite NJ. Analysis of chromatin texture by Pinkus' approximate entropy. Cytometry. Part A 2004b;59A:63.

18. Oberholzer M, Heitz PU, Kloppel G, Ehrsam RE. Morphometry in endocrine pathology. Pathol Res Pract. 1984;179(2):220-4.

19. Murata S, Mochizuki K, Nakazawa T, Kondo T, Nakamura N, Yamashita $\mathrm{H}$, et al. Morphological abstraction of thyroid tumor cell nuclei using morphometry with factor analysis. Microsc ResTech. 2003;61(5):457-62

20. Gupta N, Sarkar C, Singh R, Karak AK. Evaluation of diagnostic efficiency of computerized image analysis based quantitative nuclear parameters in papillary and follicular thyroid tumors using paraffin-embedded tissue sections. Pathol Oncol Res. 2001;7(1):46-55.

21. Wouver GVD, Weyn B, Scheunders P, Jacob W, Marck EV, Dyck DV. Wavelets as chromatin texture descriptors for the automated identification of neoplastic nuclei. J Microsc. 2000;197(Pt 1):25-35.

22. Shaposhnikov VM. Combined morphologic criteria in the diagnosis of thyroid diseases. Arkh Patol. 1991;53(1):23-7.

23. Mello MR, Metze K, Adam RL, Pereira FG, Magalhães MG, Machado CG, et al. Phenotypic subtypes of acute lymphoblastic leukemia associated with different nuclear chromatin texture. Anal Quant Cytol Histol. 2008;30(2):92-8.

24. Metze K, Ferreira RC, Adam RL, Leite NJ, Ward LS, de Matos PS. Chromatin texture is size dependent in follicular adenomas but not in hyperplastic nodules of the thyroid. World J Surg. 2008;32(12):2744-6.

25. Kayser K, Kayser G, Metze K. The concept of structural entropy in tissue-based diagnosis. Anal Quant Cytol Histol. 2007;29(5): 296-308.

26. Auada MP, Adam RL, Leite NJ, Puzzi MB, Cintra ML, Rizzo WB, et al. Anal Quant Cytol Histol. Texture analysis of the epidermis based on fast Fourier transformation in Sjögren-Larsson syndrome. Cytol Histol. 2006;28(4):219-27.

27. Doudkine A, Macaulay C, Poulin N, Palcic B. Nuclear texture measurements in image cytometry. Pathologica. 1995;87(3):286-99.

28. Hirokawa M, Carney JA, Goellner JR, De Lellis RA, Heffess CS, Katoh $\mathrm{R}$, et al. Observer variation of encapsulated follicular lesions of the thyroid gland. Am J Surg Pathol. 2002;26(11):1508-14.

29. Elsheikh TM, Asa SL, Chan JK, DeLellis RA, Heffess CS, LiVolsi $\mathrm{VA}$, et al. Interobserver and intraobserver variation among experts in the diagnosis of thyroid follicular lesions with borderline nuclear features of papillary carcinoma. Am J Clin Pathol. 2008;130(5):736-44.

30. Crissman JD, Drozdowicz S, Johnson C, Kini SR. Fine needle aspiration diagnosis of hyperplastic and neoplastic follicular nodules of the thyroid. Anal Quant Cytol Histol. 1991;13(5):321-8. 